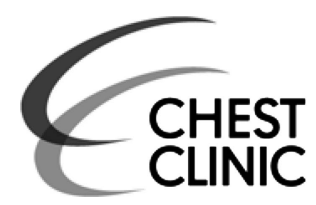
CHEST

${ }^{1}$ Division of Epidemiology and Public Health, UK Centre for Tobacco and Alcohol Studies, University of Nottingham, Clinical Sciences Building, Nottingham City Hospital, Nottingham, UK

${ }^{2}$ Department of Infection, Immunity and Inflammation, Institute for Lung Health, Department of Respiratory Medicine, Glenfield Hospital, Leicester, UK

\section{Correspondence to} Dr Lisa Szatkowski, Division of Epidemiology and Public Health, UK Centre for Tobacco and Alcohol Studies, University of Nottingham, Clinical Sciences Building, Nottingham City Hospital, Nottingham NG5 1PB, UK; lisa.szatkowski@ nottingham.ac.uk

Received 5 September 2014 Accepted 25 September 2014 Published Online First 10 November 2014

\section{CrossMark}

To cite: Szatkowski $L$, Murray $R$, Hubbard $R$, et al. Thorax 2015;70:498-500.

\title{
Prevalence of smoking among patients treated in NHS hospitals in England in 2010/2011: a national audit
}

\author{
L Szatkowski, ${ }^{1}$ R Murray, ${ }^{1}$ R Hubbard, ${ }^{1}$ S Agrawal, ${ }^{2}$ Y Huang, ${ }^{1}$ J Britton ${ }^{1}$
}

\section{ABSTRACT}

Using data from The Health Improvement Network and Hospital Episode Statistics, we investigate smoking prevalence, number of smokers treated and opportunities for cessation intervention among patients treated in National Health Service (NHS) hospitals in England from April 2010 to March 2011. Our results show that approximately 1.1 million smokers are treated in English hospitals each year, receiving a total of 2.6 million episodes of care. These findings suggest that delivering smoking cessation as a routine component of hospital care, as recommended by recent National Institute for Health and Care Excellence guidance, could achieve marked reductions in the prevalence of smoking and improve the cost-effectiveness of NHS hospitals.

\section{INTRODUCTION}

Smoking was responsible for over 460000 adult admissions to National Health Service (NHS) hospitals in England in the year to March 2012, and treating diseases caused by smoking cost the NHS over $£ 5$ billion, about $5 \%$ of its annual budget, in the 2005-2006 financial year. ${ }^{1}$ Since smoking is entirely preventable and treatable, many hospital admissions represent an avoidable drain on scarce financial and wider NHS resources.

Treating smoking should be a high priority in the delivery of NHS services; however, the proportion of smokers who receive treatment for smoking from the NHS each year is extremely low and falling. ${ }^{2}$ Contrary to Domain 1 of the NHS Outcomes Framework to prevent people dying prematurely, this low engagement with smokers represents a failure by the NHS to treat the biggest avoidable cause of disease, death and social inequality in health in the UK. Since delivering smoking cessation interventions in secondary care is highly cost-effective, ${ }^{3}$ it also reflects a failure to manage NHS budgets effectively by prioritising the most cost-effective activities.

Guidance issued by the National Institute for Health and Care Excellence (NICE) in 2013 recommended the routine and systematic delivery of cessation support to all smokers using secondary care services. ${ }^{3}$ However, the prevalence of smoking among those treated in NHS hospitals, and hence the number of people who might benefit, is unknown. This audit was therefore designed to estimate the prevalence of smoking among NHS hospital inpatients in England, the number of smokers treated and the number of potential opportunities for smoking cessation, by treatment speciality in the year 2010-2011.

\section{METHODS}

We identified all patients aged 15 and over who were registered with one of 72 general practices contributing to The Health Improvement Network (THIN) database for the year from 1 April 2010 to 31 March 2011, and used linked Hospital Episode Statistics (HES) data to determine who of these experienced one or more finished consultant episodes (FCEs) of hospital care. For those with more than one FCE during the study year, we selected an episode at random, identified the treatment specialty and took the admission date of the FCE as the index date on which to assess smoking status. For patients with no FCEs, we assigned a random index date during the study year.

Smoking status at the index date (current, ex, never or unknown) was taken to be the most recent recorded in the general practitioner (GP) record, up to 27 months before this date, in line with the Quality and Outcomes Framework (QOF) requirement for GPs to ascertain smoking status in all patients aged 15 and over at least once every 27 months. Since the QOF gives practices 90 days in which to record the smoking status of new patients, we excluded any patients who at the index date had less than 90 days of recorded GP data.

We quantified the proportions of current and ex-smokers among patients who did and did not have an FCE during the study period, and among those who did, by clinical specialty of the index FCE. To account for demographic differences between patients treated in each speciality, we calculated the relative odds and 95\% CIs of being a current smoker among those treated in any given speciality and overall, compared with those without an FCE, with adjustment for age and sex.

We used our prevalence estimates and Office for National Statistics mid-2010 national population data to estimate the number of smokers aged $15+$ treated as inpatients in 2010/11 in England as a whole. Finally, we used data on the number of FCEs in England by speciality ${ }^{4}$ for patients aged $15+$ in $2010 / 2011$ to estimate the number of FCEs nationally in which the patient was a current or ex-smoker and thereby the number of opportunities for delivering cessation interventions.

Data analysis was carried out using Stata V.12 (Stata Corp, College Station, Texas, USA). Ethics 
approval was granted by THIN Scientific Review Committee (reference 12-025).

\section{RESULTS}

There were 551320 patients aged $15+$ registered in the 72 practices in the study year; we excluded 1342 patients with less than 90 days' data before their index date. Of those remaining, $80007(14.5 \%)$ experienced 172537 FCEs in total delivered over 151342 inpatient spells. Of patients treated in hospital, $66.9 \%$ had just one inpatient spell during the study year, $19.4 \%$ had two, and $13.7 \%$ had three or more.
A record of smoking status was available for $61.4 \%$ of patients without an FCE and $74.4 \%$ of patients with one or more. Of patients with no FCE in the study year, 14.7\% were current and 19.8\% ex-smokers; in those with at least one FCE, these figures were $17.0 \%$ and $30.2 \%$, respectively. When compared by treatment specialty, the prevalence of ever (current or ex) smoking was generally similar across all specialties but highest in those treated for mental illness; however, the prevalence of current smoking was over twice as high in those with mental illness compared with other specialities. The age and sex-adjusted odds of being a current smoker, relative to those not treated in hospital, were increased by a ratio of $1.36(95 \%$

Table 1 Smoking status by speciality, adjusted ORs relative to patients not treated, and estimated number of national FCEs for patients aged $15+$ in England in 2010/2011 in current and ex-smokers (ranked by \% current smokers)

\begin{tabular}{|c|c|c|c|c|c|c|c|}
\hline Speciality & $\begin{array}{l}\text { Number of } \\
\text { patients in } \\
\text { study dataset }\end{array}$ & $\begin{array}{l}\% \text { current } \\
\text { smokers }\end{array}$ & $\begin{array}{l}\% \text { ex } \\
\text { smokers }\end{array}$ & $\begin{array}{l}\text { Adjusted OR for current } \\
\text { smoking, } 95 \% \mathrm{Cl} \text {, p value* }\end{array}$ & $\begin{array}{l}\text { Total number of } \\
\text { FCEs in patients } \\
\text { aged } 15+t\end{array}$ & $\begin{array}{l}\text { Number of } \\
\text { episodes where } \\
\text { patient current } \\
\text { smoker } ¥\end{array}$ & $\begin{array}{l}\text { Number of } \\
\text { episodes where } \\
\text { patient ex-smoker§ }\end{array}$ \\
\hline Not admitted & 469971 & 14.7 & 19.8 & Baseline & & & \\
\hline Admitted & 80007 & 17.0 & 30.2 & 1.36 (1.33 to 1.39$) p<0.001$ & 15218526 & 2591869 & 4590267 \\
\hline $\begin{array}{l}\text { Adult mental } \\
\text { illness }\end{array}$ & 472 & 51.7 & 10.6 & 5.40 (4.50 to 6.48$) p<0.001$ & 121363 & 62739 & 12856 \\
\hline Neurology & 435 & 23.2 & 28.0 & 1.83 (1.46 to 2.29$) p<0.001$ & 110457 & 25646 & 30979 \\
\hline Oral surgery & 1501 & 22.3 & 20.7 & 1.55 (1.37 to 1.75$) p<0.001$ & 185189 & 41208 & 38247 \\
\hline Thoracic medicine & 1178 & 21.9 & 37.4 & 2.06 (1.79 to 2.37$) p<0.001$ & 328631 & 71975 & 122748 \\
\hline Neurosurgery & 514 & 21.6 & 28.6 & 1.71 (1.39 to 2.12$) p<0.001$ & 79511 & 17171 & 22740 \\
\hline Pain management & 880 & 21.6 & 29.3 & 1.88 (1.60 to 2.21) $p<0.001$ & & & \\
\hline $\begin{array}{l}\text { Accident and } \\
\text { emergency }\end{array}$ & 3320 & 21.5 & 26.5 & 1.80 (1.65 to 1.96$) p<0.001$ & 616188 & 132703 & 163141 \\
\hline $\begin{array}{l}\text { Ear, nose and } \\
\text { throat }\end{array}$ & 2076 & 21.5 & 25.3 & $1.64(1.47$ to 1.82$) p<0.001$ & 255163 & 54818 & 64651 \\
\hline Vascular surgery & 440 & 20.5 & 38.2 & 1.88 (1.49 to 2.37$) p<0.001$ & & & \\
\hline Upper GI surgery & 402 & 20.4 & 31.1 & $1.64(1.28$ to 2.10$) p<0.001$ & & & \\
\hline Gynaecology & 5089 & 20.1 & 21.8 & 1.32 (1.23 to 1.41$) p<0.001$ & 1023518 & 205548 & 222845 \\
\hline $\begin{array}{l}\text { Maxillo-facial } \\
\text { surgery }\end{array}$ & 718 & 18.8 & 24.2 & 1.40 (1.16 to 1.70$) p<0.001$ & & & \\
\hline General medicine & 12343 & 18.7 & 32.8 & 1.67 (1.59 to 1.75$) p<0.001$ & 2807520 & 523609 & 920752 \\
\hline Obstetrics & 4960 & 18.6 & 24.8 & $0.98(0.92$ to 1.06$) p=0.667$ & 751316 & 139963 & 186163 \\
\hline General surgery & 9433 & 18.3 & 29.1 & 1.46 (1.39 to 1.54$) p<0.001$ & 1704643 & 312449 & 496412 \\
\hline Clinical oncology & 433 & 16.9 & 33.9 & $1.49(1.16$ to 1.92$) p=0.002$ & 346188 & 58364 & 117528 \\
\hline Midwifery & 1803 & 16.1 & 23.6 & $0.81(0.72$ to 0.92$) p=0.001$ & 263599 & 42398 & 62281 \\
\hline Gastroenterology & 5182 & 16.0 & 32.0 & 1.31 (1.22 to 1.41$) p<0.001$ & 755762 & 120759 & 241809 \\
\hline Plastic surgery & 1713 & 15.7 & 28.5 & $1.20(1.05$ to 1.37$) p=0.006$ & 216779 & 34042 & 61883 \\
\hline Urology & 4731 & 15.1 & 35.5 & 1.28 (1.18 to 1.39$) p<0.001$ & 791324 & 119259 & 281170 \\
\hline $\begin{array}{l}\text { Trauma and } \\
\text { orthopaedics }\end{array}$ & 10170 & 14.9 & 28.7 & $1.18(1.12$ to 1.25$) p<0.001$ & 1118975 & 167021 & 320619 \\
\hline Medical oncology & 430 & 14.9 & 33.7 & $1.32(1.01$ to 1.73$) p=0.041$ & 212779 & 31669 & 71751 \\
\hline Colorectal surgery & 1122 & 14.3 & 33.5 & 1.16 (0.98 to 1.37$) p=0.088$ & & & \\
\hline Rheumatology & 553 & 14.1 & 31.6 & $1.17(0.92$ to 1.48$) p=0.216$ & 147583 & 20816 & 46703 \\
\hline Nephrology & 405 & 14.1 & 38.0 & $1.21(0.91$ to 1.60$) p=0.189$ & 159858 & 22499 & 60786 \\
\hline Breast surgery & 475 & 13.9 & 25.9 & $1.09(0.84$ to 1.41$) p=0.533$ & & & \\
\hline Dermatology & 944 & 13.1 & 32.9 & $1.14(0.94$ to 1.38$) p=0.178$ & 115950 & 15231 & 38200 \\
\hline Cardiology & 3715 & 13.1 & 41.3 & $1.18(1.07$ to 1.30$) p=0.001$ & 571086 & 74556 & 236121 \\
\hline $\begin{array}{l}\text { Clinical } \\
\text { haematology }\end{array}$ & 669 & 11.7 & 33.3 & $0.97(0.77$ to 1.23$) \mathrm{p}=0.822$ & 396215 & 46195 & 132072 \\
\hline Geriatric medicine & 2044 & 11.2 & 35.3 & $1.10(0.95$ to 1.26$) p=0.197$ & 719805 & 80644 & 254256 \\
\hline Ophthalmology & 4586 & 9.0 & 39.9 & $0.85(0.77$ to 0.94$) p=0.002$ & 611203 & 55043 & 244161 \\
\hline
\end{tabular}

${ }^{*}$ Adjusted for sex and age group (15-19, 20-24, 25-34, 35-49, 50-59, 60+).

tData not available for all treatment specialities.

$\ddagger$ Calculated as total number of FCEs for speciality $\times$ prevalence of current smoking for speciality.

$\S C$ Calculated as total number of FCEs for speciality $\times$ prevalence of ex-smoking for speciality.

FCE, finished consultant episode; Gl, gastrointestinal. 
CI 1.33 to $1.39, \mathrm{p}<0.001)$ among those with any FCE but also varied substantially according to treatment specialty (table 1 ).

Extrapolation of our prevalence data to the total number of FCEs in England in the study year suggests that approximately 2.6 million episodes of care were delivered to current smokers in 2010-2011. There were an estimated 43318368 people aged $15+$ in England in mid-2010, and extrapolating our estimates that $14.5 \%$ of these were treated as a hospital inpatient, and that $17 \%$ of patients were smokers, indicates that during the study period approximately 2.6 million episodes of care were delivered to approximately 1.1 million individual smokers.

\section{DISCUSSION}

This study demonstrates that each year in England around 2.6 million episodes of inpatient care are delivered to approximately 1.1 million smokers. At an individual and population level, intervening to maximise the number of smokers who receive effective NHS support to stop smoking as part of their hospital care represents an opportunity for both health promotion and cost management through a reduction in demand on NHS services. This is particularly true of people treated in mental health settings, among whom current smoking rates are double those in other specialities.

THIN dataset is broadly representative of the UK population and estimates of the prevalence of current smoking from THIN are comparable with those derived from national survey data, ${ }^{5}$ adding credibility to our estimates of the burden of smoking and opportunities for intervention at a national level.

NHS Stop Smoking Services provide freely available behavioural support and pharmacotherapy, but these services are predominantly community-based and hence not immediately available to smokers admitted to or discharged from hospital. Separate funding streams for community and hospital services mitigate against service integration since both services can argue that the other is responsible for inpatient smoking cessation provision. Our own recent trial data demonstrate that treating smoking as a default at the point of admission substantially increases the proportion of smokers accepting support, ${ }^{6}$ and this approach is now recommended in NICE guidance ${ }^{3}$ and the British Thoracic Society's Recommendations for Hospital Smoking Cessation Services. ${ }^{7}$ The findings of the present study indicate that default provision of behavioural support and pharmacotherapy to smokers treated as inpatients in English hospitals has the potential to reach 1.1 million smokers each year. NICE data indicate that this approach is cost-effective for both hospitals and wider society. ${ }^{3}$ The routine provision of smoking cessation support to smokers seen as outpatients, not captured in this study of inpatient data, will further increase the number of smokers reached. Indeed, HES outpatient data indicate 61.5 million outpatient appointments were attended by patients aged 20+ in England in the 2010-2011 financial year, approximately four times the number of inpatient FCEs, substantially increasing the number of opportunities to intervene with smokers. 4

Our finding that around $47 \%$ of people treated in hospital are current or ex-smokers, compared with about $35 \%$ of those not treated, is broadly consistent with the strong role that smoking plays in causing disease, particularly respiratory and cardiovascular disease. However, the proportion of hospital patients who had quit smoking was also generally high, at around two-thirds, and broadly similar between specialties, with the exception of mental illness. Over $60 \%$ of people treated for mental illness were ever-smokers and 50\% were current smokers. People with mental health problems are more likely to be smokers and to be more heavily addicted, but the extraordinarily high prevalence of continued smoking in this group reflects the previously highlighted failure of existing approaches to engage with this patient group.

Our study thus identifies both the importance and the magnitude of the opportunity to intervene to treat smokers and reduce the prevalence of smoking in NHS secondary care users. Implementation of current NICE guidance to deliver smoking cessation interventions as a routine component of secondary care provision could have a major impact on the prevalence of smoking, and hence on the morbidity and mortality that smoking causes.

Contributors RH, SA and LS designed the study; YH extracted THIN and HES data; LS analysed the data; $L S, J B$ and RM wrote the first draft of the manuscript and all authors contributed to and have approved the final version. All authors had full access to all of the data (including statistical reports and tables) in the study and take responsibility for the integrity of the data and the accuracy of the data analysis.

\section{Funding None.}

Competing interests All authors have completed the Unified Competing Interests form at http://www.icmje.org/coi_disclosure.pdf (available on request from the corresponding author). SA reports personal fees and non-financial support from Pfizer, non-financial support from McNeil Pharmaceuticals, personal fees and non-financial support from GlaxoSmithKline, outside the submitted work. JB chaired NICE programme development group that published guidance recommending integration of smoking cessation interventions into routine secondary care provision in November 2013, and RM was a member of this programme development group.

Ethics approval Ethical approval for the use of the linked THIN-HES data was granted by THIN Scientific Review Committee (reference number 12-025).

Provenance and peer review Not commissioned; internally peer reviewed. Data sharing No additional data are available.

\section{REFERENCES}

1 The Information Centre. Statistics on smoking: England, 2013. London: The Health and Social Care Information Centre, 2013.

2 Health and Social Care Information Centre. Statistics on NHS stop smoking services: England, April 2012 to March 2013. London: Health and Social Care Information Centre, 2013.

3 National Institute for Health and Care Excellence. Smoking cessation in secondary care: acute, maternity and mental health services. NICE public health guidance 48 . London: National Institude for Health and Care Excellence, 2013.

4 Health and Social Care Information Centre. Hospital Episode Statistics.

5 Szatkowski L, Lewis S, McNeill A, et al. Can data from primary care medical records be used to monitor national smoking prevalence? J Epidemiol Community Health 2012;66:791-5.

6 Murray RL, Leonardi-Bee J, Marsh J, et al. Systematic identification and treatment of smokers by hospital based cessation practitioners in a secondary care setting: cluster randomised controlled trial. BMJ 2013;347:f4004.

7 British Thoracic Society. British thoracic society recommendations for hospital smoking Cessation services for commissioners and health care professionals. London: British Thoracic Society, 2012. 